\title{
Sensitive biomarker for assessing the effects of the environment on the population of plants
}

\author{
L.A. Minasbekyan ${ }^{1 *}$, G.S. Aidarkhanova² ${ }^{2}$ I.A. Avagyan ${ }^{3}$ \\ ${ }^{1}$ YSU, Research Institute of Biology, Yerevan, Armenia \\ ${ }^{2}$ S. Seyfulin Kazakh AgroTechnical university, Nur-Sultan, Kazakhstan \\ ${ }^{3}$ SRC of V\&TC at AM of RA, Darakert, Armenia
}

DOI 10.18699/ICG-PlantGen2019-34

(c) Autors, 2019

* e-mail:minlia@ysu.am

\begin{abstract}
In the last few years, special importance has been given to the study of the population of pasture plants, since environmental pollution through animal food can be transmitted to humans. Due to these circumstances, it is imperative to regularly monitor pasture plants, both for relocating animals to more environmentally friendly meadows and as environmental protection measures aimed at improving damaged pastures. A great number of biotic and abiotic factors affect plant growth under natural growing conditions. No DNA sequence carries the complete information necessary to determine the phenotype of the organism. DNA methylation controls genomic integration, regulates genome expression and cell differentiation as well as the plant response to biotic and abiotic stresses. Epigenetic regulation involves various reversible chemical modifications occurring on both the DNA itself and the proteins interacting with it, affecting the chromatin structure and function without, however, altering the sequence of nucleic residues in the DNA. Epigenetics holds promise to explain at least a part of the influences the environment has on plants' phenotypes. Among pasture plants are widely distributed Bromus inermis, Medicago sativa, Onobrychis arenaria, Agropyron pectinoforme, etc. We have studied DNA methylation in esparcet (Onobrichis arenaria) and crested wheat grass (Agropyron cristatum) from the Kazakh Steppe and discuss the environmental factors that can led to changes in epigenetics marks. The data obtained on epigenetic changes in the plants studied will improve the methods of pasture monitoring, taking into account the ecological, climatic conditions of the regions and the agricultural sector of the countries' economies. Below is summarized literature data-based evidence about the sensitivity of DNA methylation and its use as a biomarker for ecological epigenetics. Data sets for model plants under different abiotic factors and non-model plants established for ecological epigenetics are provided. Key words: epigenetics; DNA methylation; biomarkers; abiotic stress factors.
\end{abstract}

\section{Introduction}

Plants growth under environmental conditions in the assessed climate and landscape is constantly subject to different environmental factors whcih, when rapid and extreme, can be recognized as stresses. Among the stresses the plants are constantly confronted with are both biotic (hormones, pests and insects) and abiotic (osmtic, temperature fluctuations, drought, radiofrequency, metal and microelemens) environmental impact factors, which seriously reduce crop productivity and the quality of pasture plants. Plant responses to the abiotic stresses, such as salt, drought, extreme temperatures, and human-made extremely high frequencies, are complex and involve numerous physiological, cellular and molecular adaptations.

\section{Epigenetic changes in model plants under some abiotic stresses}

Plants have a special system to adjust themselves to extreme external stressful conditions through instantly transmitting signals. Plant cells receiving signals from external stimuli through fluctuations in cytosolic concentrations decode them using their own machinery to the secondary messenger. $\mathrm{Ca}^{2+}$ is encoded in various stimuli of abiotic and biotic stresses. Recently, the Calcineurin B-like (CBL) protein-CBL-interacting protein kinase (CIPK) complex has been widely accepted as a
$\mathrm{Ca}^{2+}$ signaling mechanism, which is involved in the response to different external stress signals, including mm-wave impact (Manik et al., 2015; Pall, 2016). Exposure of 3-week tomato seeds to a high- frequency, low-amplitude electromagnetic field leads to altered expressions of at least five stress-related genes (Vian et al., 2016).

Under different abiotic and biotic stress factors, plant experience the alteration of many processes. It is known that at least four different regulons act in response to abiotic stresses. Dehydration-responsive element binding protein 1 (DREB1)/C-repeat binding factor (CBF) acts in response to cold stress. In response to heat stress, DREB2 regulons start to act influencing ABA-independent gene expression. The ABAresponsive element (ABRE) binding protein (AREB)/ABRE binding factor (ABF0 regulon functions in ABA-dependent gene expression under osmotic stress conditions (Ciarmiello et al., 2014).

In addition, there are NAC and MYB/MYC regulons involved in stress response gene expression. Expression profiling from 23 selected T. aestivum NAC genes at the developmental stages in field drought conditions identified seven with leafspecific expression and five with a grain-specific expression, the profiles of which depended on the genotype (Guerrin et al., 2019). 
Recent studies shows a positive effect of a combination of biotic and abiotic stresses on plant performance, reducing sensitivity to biotic stress. The interaction between both types of stress points to a crosstalk between their conformable signaling pathways. Such crosstalk may be synergistic and/or antagonistic and may include, among others, the involvement of phytohormones, transcription factors, kinase cascades and reactive oxygen species, which can lead to an enhancement in plant resistance against pathogens (Rejeb et al., 2014).

Multiple studies of model plants provide evidence that, in response to different abiotic and biotic stresses, epigenetic modifications in gene expression take place, which are heritable changes and are not encoded by a DNA sequence (Yong-Villalobos et al., 2015; Yong et al., 2016; Banerjee, Roychoudhury, 2018).

Although DNA methylation is only a minor chemical modification of cytosin residues, it is an important epigenetic modification, which carries valuable information for properly regulating gene expression and, therefore, a broad range of biological processes and diseases. DNA methylation is tissue-specific, dynamic, sequence-context-dependent and trans-generationally heritable, and these complex patterns of methylation highlight the significance of profiling DNA methylation to answer biological questions (Yong et al., 2016). Epigenetic modifications in the model plant characterized by genome-wide DNA methylation, histone modification, histone variant deposition, various classes of small RNAs, nucleosomal positioning, and chromatin modifications have a transgenerational adaptive response to biotic and abiotic environmental stresses (Dukowic-Shulz et al., 2018; Kumar et al., 2018; Wakeel et al., 2018; Weinhold, 2018).

\section{Epigenetic alterations in non-model plants}

The ecological and evolutionary significance of natural epigenetic variation commonly depends critically on whether epigenetic states are transmitted from parents to offspring. Although little is known about epigenetic inheritance in non-model plants, it has been shown that DNA methylation mediates the inherited genotype-specific effects of drought stress in P. persicaria (Herman, 2016).

The study showed that the genotype, epigenotype, and parental soil-moisture environment interact with systems, including drought, nutrient limitation, pathogen infection, high salinity, temperature shock, and UV radiation. Such DNA methylation changes have been associated with the induced expression of critical stress-response genes, thus implicating DNA methylation in the expression of adaptive phenotypic plasticity (Herman, 2016). The extensive transgenerational transmission of genome-wide global cytosine methylation and anonymous epigenetic markers in the non-model species L. latifollia was revealed (Herrera et al., 2017).

Analysis of methylation-sensitive amplification polymorphism (MSAP) has been often used to assess methyl-cytosine changes in response to stress treatment and, quite lately, in ecological studies of wild plant populations. The paper summarizes literature data on global DNA methylation in wild plants populations, analyzes the relationship between MSAP results and the percentage of global cytosine methylation in genomic DNA obtained by HPLC analysis (Alonso et al.,
2016). Notably, that environmentally induced epigenetic effects may be either transient or persistent across generations, and heritable changes may be either selected or linked to something that is selected. Future analyses should consider that part of the epigenetic variation is similar to phenotypic variation, and carefully designed experiments are necessary to characterize both genetic and environmental contributions to epigenetic variation (Richards et al., 2017).

\section{Conclusion}

The obtained data on epigenetic changes of the pasture plants will improve the methods of monitoring pastures used for agricultural purposes, taking into account the ecological, climatic conditions of the regions and the agricultural sector of the countries' economies.

The results of such studies will help to rank the populations of forage plants according to the rate of formation of microevolutions and will contribute to the improvement of traditional methods of agricultural technology. For natural pastures, one must also take into account the fact that the most important factor leading to the dynamical epigenetic changes in the populations of wild plants is climate change.

\section{References}

Alonso C., Perez R., Bazaga P., Medrano M., Herrera C.M. MSAP markers and global cytosine methylation in plants: a literature survey and comparative analysis for a wild-growing species. Mol. Ecol. Resources. 2016;16:80-90.

Banerjee A., Raychoudhary A. The gymnastics of epigenomics in rice. Plant Cell Rep. 2018;37:25-49. https://doi.org/10.107/s00299-0172192-2.

Ciarmiello L.F., Woodrow P., Piccirillo P., De Luca A., Carillo P.Ch. 3 "Transcription Factors and Environmental Stresses in Plants". In: Emerging Technologies and Management of Crop Stress Tolerance. 2014;57-78. ISBN: 9780128008768, https://doi.org/10.1016/b9780-12-800876-8.00003-5.

Dukowic-Schulze S., Liu C., Chen C. Not just expression: 3D implications of chromatin modifications during sexual plant reproduction. Plant Cell Rep. 2018;37:11-16. DOI 10.1007/s00299-017-2222-0.

Guerrin C., Roche J., Allard V., Ravel C., Mouzeyar S., Bouzidi M.F. Genome-wide analysis, expansion and expression of the NAC family under drought and heat stresses in bread wheat (T. aestivum L.). PLoS ONE. 2019;14(3):e0213390. https://doi.org/10.1371/journal. pone. 0213390

Herman J.J. Epigenetics of adaptive plasticity: an investigation of plant responses to environmental stress within and across generations. Diss. on PhD, Wesleyan University, 2016.

Herrera C.M., Alonso C., Medrano M., Perez R., Bazaga P. Transgenerational epigenetics: inheritance of global cytosine methylation and methylation-related epigenetic markers in the shrub Lavandulalatifolia. American J. Bot. 2017;105(4):1-8. DOI 10.1002/ajb2. 1074.

Kumar V., Khare T., Shriram V., Wani S.H. Plant small RNAs: the essential epigenetic regulators of gene expression for salt-stress responses and tolerance. Plant Cell Rep. 2018;37:61-75 https://doi. org/10.1007/s00299-017-2210-4.

Manik N.S.M., Shi S., Mao J., Dong L., Su Y., Wang Q., Liu H. The calcium sensor CBL-CIPK is involved in plant's response to abiotic stresses. Int. J. Genomics. 2015; article ID 493191.

Pall M.L. Electromagnetic fields act similarity in plants as in animals: probable activation of calcium channels via thei. Current Chemical Biol. 2016;10(1):74-82. DOI 10.2174/ 22127968106661 60419160433 
Rejeb I.B., Pator V., Mauch-Mani B. Plant response to simultaneous biotic and abiotic stress: molecular mechanisms. Plants. 2014;458475. DOI 10.3390/plants3040458.

Richards C.L., Alonso C., Becker C., Bossdorf O. et al. Ecological plant epigenetics: evidence from model and non-model species, and the way forward. Ecol. Letters. 2017;20:1576-1590. DOI 10.1111/ele. 12858

Vian A., Davies E., Gendraud M., Bonnet P. Plant responses to high frequency electromagnetic fields. Bio Med Research International. 2016; Article ID 1830262.

Wakeel A., Ali I., Khan A.R., Wu M., Upretti S., Liu D, Liu B., Gan Y. Involvement of histone acetylation and deacetylation in regulating auxin responses and associated phenotypic changes in plants. Plant Cell Rep. 2018;37:51-59. https://doi.org/10.1007/s00299-0172205-1.
Weinhold A. Transgenerational stress-adaption: an opportunity for ecological epigenetics. Plant Cell Rep. 2018;37:3-9. DOI 10.1007/ s00299-017-2216-y.

Yong W.-Sh., Hsu F.-M., Chen P.-Y. Profiling genome-wide DNA methylation epigenetics chromatin. 2016;9:26. DOI 10.1186/s13072016-0075-3.

Yong-Villalobos L., González-Morales S.I., Wrobel K., Gutiérrez-Alanis D., Cervantes-Peréz S.A., Hayano-Kanashiro C., Oropeza-Aburto A., Cruz-Ramírez A., Martínez O., Herrera-Estrella L. Methylome analysis reveals an important role for epigenetic changes in the regulation of the Arabidopsis response to phosphate starvation. Proc Natl Acad Sci USA. 2015;29;112(52):E7293-302. DOI 10.1073/ pnas. 1522301112.

Conflict of interest. The authors declare no conflict of interest. 